Kardiologe 2021 $15: 115-120$

https://doi.org/10.1007/s12181-021-00453-0

Angenommen: 21. Januar 2021

Online publiziert: 24. Februar 2021

(c) Deutsche Gesellschaft für Kardiologie -

Herz- und Kreislaufforschung e.V. Published by

Springer Medizin Verlag GmbH, ein Teil von

Springer Nature - all rights reserved 2021
Die Elektrophysiologie ist seit jeher geprägt von technischem Fortschritt und Innovationen. Die Suche nach effektiveren Ablations- sowie Visualisierungsmöglichkeiten beschäftigt und begleitet die Elektrophysiologie nun seit vielen Jahren. Von der reinen Röntgendurchleuchtung hin zur multimodalen Bildintegration und zum hochauflösenden 3-D-Mapping war und ist die Elektrophysiologie geprägt von technischen Innovationen, die über Jahre hinweg zu immer größerer Patientensicherheit, besserem Verständnis der Arrhythmiemechanismen sowie zur erfolgreicheren Therapie beigetragen haben.

Aber nicht nur die reine technische Innovation, sondern insbesondere die wissenschaftliche Evaluation neuer Technologien und Therapieansätze beschäftigen Jahr für Jahr die elektrophysiologische Gemeinschaft, und so stand neben vielen technischen Innovationen und neuen Ablationstechnologien das Jahr 2020 mit dem EAST-AFNET4-, dem STOP AFund EARLY AF-Trial sowie einer neuen Leitlinie der ESC (European Society of Cardiology) aus Sicht der Elektrophysiologie v. a. im Zeichen der Vorhofflimmertherapie.

\section{Stehen wir vor einem Paradigmenwechsel in der Vorhofflimmertherapie?}

Auch wenn Vorhofflimmern trotz effektiver Antikoagulation und reduzierter Symptomatik bei Frequenzkontrolle $[1,2]$ mit einer erhöhten Wahrscheinlichkeit für kardiovaskuläre Ereignisse (Tod, Schlaganfall, Herzinsuffizienz) einhergeht [3], konnte bislang im Um-

\author{
Jan-Hendrik van den Bruck' · Daniel Steven ${ }^{1}$ Philipp Sommer ${ }^{2}$ \\ ${ }^{1}$ Herzzentrum, Abteilung für Elektrophysiologie, Klinikum der Universität zu Köln, Köln, Deutschland \\ ${ }^{2}$ Klinik für Elektrophysiologie und Rhythmologie, Herz- und Diabeteszentrum NRW, Ruhr-Universität \\ Bochum, Bad Oeynhausen, Deutschland
}

\title{
Elektrophysiologie im Jahr 2020 - Ein Rückblick
}

kehrschluss für die rhythmuserhaltende Therapie kein prognostischer Vorteil nachgewiesen werden.

In der AFFIRM-Studie, die im Jahr 2002 veröffentlicht wurde und in der 4060 Patienten auf Rhythmus- oder Frequenzkontrolle randomisiert untersucht wurden, zeigte sich kein prognostischer Vorteil für den rhythmuserhaltenden Therapieansatz [4]. Daher ist die rhythmuserhaltende Therapie auch heute noch primär dem symptomatischen Patienten vorbehalten. Auch aktuelle Studien wie der CABANA-Trial (publiziert 2019) verfehlten in der entscheidenden Intention-to-Treat-Analyse den Nachweis eines prognostischen Vorteils für den Erhalt des Sinusrhythmus im Hinblick auf harte klinische Endpunkte wir Tod, Rehospitalisierung und das Auftreten von Schlaganfällen [5].

In der 2020 vorgestellten EASTAFNET4(Early Rhythm-Control Therapy in Patients with Atrial Fibrillation)Studie [6] wurden in 135 Zentren insgesamt 2789 Patienten, die entweder über 75 Jahre alt waren oder unter kardiovaskulären Begleiterkrankungen litten, untersucht. Der primäre kombinierte Endpunkt setzte sich zusammen aus kardiovaskulärem Tod, Schlaganfall oder Hospitalisierung aufgrund von akutem Koronarsyndrom oder klinischer Verschlechterung einer Herzinsuffizienz. Den sekundären Endpunkt markierte die Anzahl der im Krankenhaus verbrachten Nächte. Sehr frühzeitig nach Diagnosestellung des Vorhofflimmerns (Median 36 Tage nach Erstdiagnose) wurden die Patienten auf „usual care“ (orale Antikoagulation nach $\mathrm{CHA}_{2} \mathrm{DS}_{2} \mathrm{VASc}$, optimale Therapie der kardiovaskulären
Begleiterkrankungen) oder frühzeitige Rhythmuskontrolle randomisiert. Im Rhythmuskontrollarm wurden initial $86,8 \%$ der Patienten mit einer medikamentösen antiarrhythmischen Therapie behandelt (überwiegend Klasse Ic und Amiodaron). Innerhalb der ersten 2 Jahre wurden insgesamt lediglich 19,4\% des Kollektivs einer Katheterablation zugeführt. Diese frühzeitige Einleitung einer Sinusrhythmus-erhaltenden Therapie führte zu einer signifikanten Reduktion des Auftretens eines primären OutcomeEvents mit einer Reduktion des relativen Risikos für ein kardiovaskuläres Event um 21 \% (Hazard Ratio 0,79; $96 \%$-Konfidenzintervall $0,66-0,94 ; p=0,005)$. Im Hinblick auf die „Nächte, verbracht im Krankenaus" ergab sich kein signifikanter Unterschied zwischen beiden Gruppen $(5,8 \pm 21,9$ vs. $5,1 \pm 15,5 ; p=0,23)[6]$. Erstmalig konnten die Autoren hiermit durch frühzeitigen Therapiebeginn einen prognostischen Nutzen der rhythmuserhaltenden Therapie bei Patienten mit Vorhofflimmern und kardiovaskulären Begleiterkrankungen zeigen.

Neben der Frage nach prognostischer Relevanz der Therapie stellt sich im zweiten Schritt natürlich die Frage nach der optimalen Therapiemethode sowie des Behandlungszeitpunktes. Die Katheterablation war bisher sowohl in vergleichenden Studien als auch in den Leitlinienempfehlungen primär Patienten mit gescheitertem medikamentös antiarrhythmischem Therapieversuch vorbehalten.

Zwei nun zeitgleich im New England Journal of Medicine veröffentlichte randomisierte Studien verglichen die Vorhofflimmerablation mittels Kryoablation 


\begin{tabular}{|c|c|c|}
\hline Studie & Hauptaussage & Journal, Jahr \\
\hline $\begin{array}{l}\text { EARLY-AF } \\
\text { [7] }\end{array}$ & $\begin{array}{l}\text { Kryoablation ist als Erstlinientherapie von symptomatischem par- } \\
\text { oxysmalem Vorhofflimmern der medikamentösen Rezidivprophyla- } \\
\text { xe überlegen }\end{array}$ & NEJM, 2020 \\
\hline $\begin{array}{l}\text { EAST- } \\
\text { AFNET4 } \\
{[6]}\end{array}$ & $\begin{array}{l}\text { Frühzeitige Rhythmuskontrolle führt zu signifikanter Reduktion der } \\
\text { kardiovaskulären Ereignisrate ohne relevant zusätzliche Hospitalisie- } \\
\text { rung }\end{array}$ & NEJM, 2020 \\
\hline $\begin{array}{l}\text { STOP AF } \\
{[8]}\end{array}$ & $\begin{array}{l}\text { Kryoablation ist als Erstlinientherapie von symptomatischem par- } \\
\text { oxysmalem Vorhofflimmern der medikamentösen Rezidivprophyla- } \\
\text { xe überlegen }\end{array}$ & NEJM, 2020 \\
\hline $\begin{array}{l}\text { BERLIN-VT } \\
{[10]}\end{array}$ & $\begin{array}{l}\text { Frühzeitige VT-Ablation, vor ICD-Implantation, ohne Zusatznutzen } \\
\text { im Hinblick auf Mortalität und Hospitalisierung }\end{array}$ & $\begin{array}{l}\text { Circulation, } \\
2020\end{array}$ \\
\hline \multicolumn{3}{|c|}{$\begin{array}{l}V T \text { ventrikuläre Tachykardie, ICD implantierbarer Kardioverter-Defibrillator, NEJM New England Journal } \\
\text { of Medicine }\end{array}$} \\
\hline
\end{tabular}

als Erstlinientherapie mit einem initial medikamentös antiarrhythmischen Therapieansatz. Beide Studien untersuchten hierbei primär die Effektivität der jeweiligen Therapie.

In die EARLY-AF(Cryoablation or Drug Therapy for initial Treatment of Atrial Fibrillation)-Studie [7] wurden insgesamt 303 Patienten mit symptomatischem paroxysmalem Vorhofflimmern eingeschlossen. Die Patienten waren im Mittel ca. 60 Jahre alt mit erhaltener linksventrikulärer Pumpfunktion. Zum Monitoring des Therapieerfolgs erhielten alle Studienteilnehmer einen implantierten Eventrekorder. Nachbeobachtungszeitraum waren 12 Monate. Den primären Endpunkt markierte das erste tachyarrhythmische Rezidivereignis, und die sekundären Endpunkte bildeten u.a. Vorhofflimmer-Burden und Lebensqualität.

Nach 1 Jahr kam es mit 42,9\% in der Ablationsgruppe im Vergleich zu 67,8\% in der Medikamentengruppe zu signifikant weniger Rezidiven nach Vorhofflimmerablation. Dies führt damit zu einer Reduktion des relativen Risikos für ein Rezidiv um $52 \%$ (0,48; $95 \%$-Konfidenzintervall $0,35-0,66 ; p<0,001)$ nach frühzeitiger Kryoablation. Auch im Hinblick auf eine Verbesserung der Lebensqualität zeigte sich die Ablationsstrategie überlegen [7].

In die sehr ähnliche STOP AF(Cryoballoon Ablation as Initial Therapy for Atrial Fibrillation)-Studie [8] wurden insgesamt 203 Patienten, ebenfalls mit symptomatischem paroxysmalen Vorhofflimmern eingeschlossen. Auch der
Ausgang der STOP AF-Studie deckte sich mit den Ergebnissen des EARLYAF-Trials: Die Katheterablation ist, auch als Erstlinientherapie im Vergleich zur medikamentös antiarrhythmischen Therapie das deutlich effektivere Therapieverfahren. Bei sehr ähnlichem Patientenkollektiv zeigte sich hier nach $12 \mathrm{Mo-}$ naten Rezidivfreiheit bei $74,6 \%$ in der Ablationsgruppe verglichen mit $45 \%$ in der Medikamentengruppe (95\%-Konfidenzintervall $34,6-54,7 ; \quad p=<0,001)$ [8].

Zusammenfassend lieferten diese $3 \mathrm{im}$ letzten Jahr erschienenen Landmark-Studien neue Evidenz für die Behandlung von Vorhofflimmern, die einen nachhaltigen Einfluss auf zukünftige Therapieempfehlung haben könnte: Die Katheterablation erwies sich als das überlegene Verfahren in der Erstlinientherapie von symptomatischem paroxysmalem Vorhofflimmern, und die frühzeitige rhythmuskontrollierende Behandlung geht mit einem prognostischen Nutzen für Patienten mit kardiovaskulären Begleiterkrankungen einher. Auch wenn es sich bei EAST AFNET4 nicht um eine Ablationsstudie handelt, werfen die beiden standardisierten Kryostudien in diesem Kontext die Frage nach einer Neubewertung der Vorhofflimmertherapie auf. Bei der heutigen in den Leitlinien erfolgten Aufwertung der Ablationstherapie sind die Ergebnisse der hier besprochenen Studien noch nicht berücksichtigt. Zukünftige Studien müssen zeigen, ob die Rhythmuskontrolle mittels Katheterablation ebenfalls in der Lage ist, diese klinischen Endpunkte zu beeinflussen.

\section{Neue Leitlinien für die Diagnostik und Therapie von Vorhofflimmern}

Neben wichtigen klinischen Studien (๑Tab. 1) wurde im vergangenen Jahr eine neue europäische Leitlinie zur Diagnostik und Therapie von Vorhofflimmern veröffentlicht [9].

Ein neu gestalteter Schlagwortleitfaden, das „CC to ABC“, soll stringent von der Diagnose („Confirm“) über eine ausführliche Charakterisierung hin zu einer multimodalen, auf 3 Säulen (Antikoagulation, Bessere Symptomkontrolle und Therapie der Comorbiditäten) basierenden Therapie führen.

Ein besonderer Schwerpunkt wird hierbei auf die Charakterisierung des Vorhofflimmerns gelegt. Empfohlen wir eine Charakterisierung nach dem sog. „4S-Vorhofflimmer-Schema“. Dies beinhaltet neben Evaluation von Schlaganfallrisiko (z. B. $\mathrm{CHA}_{2} \mathrm{DS}_{2}$ VASc-Score), Schwere der Symptome (z.B. EHRASymptom-Score) und Schwere des Vorhofflimmer-Burdens (z.B. paroxysmal vs. persistierend, Episodendauer etc.) nun auch die Evaluation eines möglichen zugrunde liegenden atrialen Substrats. Anhand z. B. von Komorbiditäten, Vorhofgröße, aber auch durch Bildgebung sollte die Schwere eines möglichen arrhythmogenen atrialen Substrats evaluiert und anschließend als prognostischer Parameter zur Planung des weiteren therapeutischen Prozedere herangezogen werden.

\section{》) Bezüglich der Therapie betonen die neuen Leitlinien die Bedeutung eines multimodalen Therapieansatzes}

Bezüglich der Therapie betonen die neuen Leitlinien die Bedeutung eines multimodalen Therapieansatzes. Ein zunehmender Stellenwert wird hier der Therapie modifizierbarer Risikofaktoren, insbesondere der Gewichtsreduktion bei Übergewicht (Ziel-BMI [Body-MassIndex] $<27 \mathrm{~kg} / \mathrm{m}^{2}$ ), sowie Lebensstilmodifikationen eingeräumt. 
Darüber hinaus nimmt der Stellenwert der Rhythmuskontrolle mit einer nun abgegebenen Klasse-IA-Empfehlung zur rhythmuserhaltenden Therapie bei bestimmten Patienten weiter zu.

Bei Betrachtung der Rhythmuskontrollstrategie wurde die Indikation für die katheterinterventionelle Behandlung des Vorhofflimmerns ausgeweitet: Während sich die Empfehlung für die Erstlinientherapie (noch) nicht geändert hat, liegt nun nach frustranem medikamentösem Therapieversuch nicht nur für paroxysmales Vorhofflimmern, sondern auch für persistierendes Vorhofflimmern eine Klasse-I-Empfehlung für die Vorhofflimmerablation vor. Neuerungen gibt es ebenfalls für die Therapie von Vorhofflimmern bei Patienten mit eingeschränkter linksventrikulärer (LV) Pumpfunktion: Bei Verdacht auf eine tachymyopathische Genese der reduzierten LV-Funktion empfehlen die aktualisierten Leitlinien eine interventionelle Therapie, unabhängig des Vorliegens von Symptomen, als Erstlinientherapie mit einem Klasse-I-Empfehlungsgrad. Neu ist in diesem Zusammenhang auch die Empfehlung der Katheterablation aus prognostischen Gesichtspunkten (Klasse IIa) in diesem Patientenkollektiv [9]. Im Focus für die Zukunft steht somit neben zunehmender Bedeutung der Katheterablation in der Vorhofflimmertherapie v. a. die Notwendigkeit patientenindividualisierter Behandlungsansätze und Therapieplanung.

\section{Neue Ablationstechniken - "high power short duration"}

Die Suche nach der optimalen Ablationstechnik stellt seit jeher einen der wichtigsten Antriebe für Innovationen in der Elektrophysiologie dar. Ein optimales Ablationsverfahren führt innerhalb kürzester Zeit zu einer effektiven transmuralen Läsion und beschränkt hierbei den Zellschaden auf die Zielregion. Betrachtet man die physikalische Wirkung des Hochfrequenzstroms im Gewebe, unterscheidet man die eher oberflächliche Widerstandserwärmung zu Beginn eines Ablationsimpulses und den anschließenden konduktiven Wärmetransfer in die tieferen Gewebeschichten bei

Kardiologe 2021 · 15:115-120 https://doi.org/10.1007/s12181-021-00453-0

(c) Deutsche Gesellschaft für Kardiologie - Herz- und Kreislaufforschung e.V. Published by Springer Medizin Verlag GmbH, ein Teil von Springer Nature - all rights reserved 2021

\section{J.-H. van den Bruck · D. Steven · P. Sommer \\ Elektrophysiologie im Jahr 2020 - Ein Rückblick}

\section{Zusammenfassung}

Wir blicken zurück auf ein ereignisreiches Jahr 2020, das aus elektrophysiologischer Sicht mit 3 Landmark-Studien und einer neuen Leitlinie ganz im Zeichen der Vorhofflimmertherapie stand. Die mit Spannung erwarteten Ergebnisse der EASTAFNET4-Studie zeigten, dass die frühzeitige Einleitung einer rhythmuserhaltenden Therapie zu einer signifikanten Reduktion der kardiovaskulären Ereignisrate (Schlaganfall, Hospitalisierung bei Herzinsuffizienz oder akutem Koronarsyndrom, kardiovaskulärer Tod) führt, ohne dass hierfür eine nennenswerte zusätzliche Hospitalisierung notwendig ist. Erstmalig konnte somit ein prognostischer Nutzen der rhythmuserhaltenden Therapie nachgewiesen werden.
Darüber hinaus unterstrichen die EARLY-AFund STOP AF-Studien den Stellenwert der Katheterablation als effektives Mittel zum Erhalt des Sinusrhythmus. Der zunehmende Stellenwert der Katheterablation findet sich ebenso wie die steigende Relevanz individualisierter Behandlungskonzepte und Substratcharakterisierung in der neuen Leitlinie wieder. Abschließend erlangten im vergangenen Jahr aussichtsreiche neue Ablationstechniken die Marktreife und versprechen für das kommende Jahr zusätzliche spannende Erkenntnisse.

Schlüsselwörter

Vorhofflimmerablation - Klinische Studien . Leitlinie · Innovationen · Katheterablation

\section{Electrophysiology in 2020-A review}

\begin{abstract}
We look back on an eventful year 2020, which from an electrophysiological perspective stood out with three landmark studies and the new guidelines as an important year for the treatment of atrial fibrillation. The results of the EAST-AFNET4 trial demonstrated that early initiation of rhythm control is associated with a significant reduction in the rate of cardiovascular events (stroke, hospitalization with heart failure or acute coronary syndrome, death from cardiovascular causes) without requiring additional hospitalization. Hence, a prognostic benefit could be shown for the first time for rhythm control strategy. Furthermore, the EARLY-AF and STOP AF
\end{abstract}

trials underlined the importance of catheter ablation as an effective means to maintain sinus rhythm. The new 2020 guidelines moreover emphasize the significance of individualized treatment concepts, substrate characterization and the role of catheter ablation in the treatment of atrial fibrillation. Finally, several new ablation techniques have become available for clinical use, which gives the prospect of another exciting year in electrophysiology.

Keywords

Atrial fibrillation ablation - Clinical trials . Guidelines · Innovations - Catheter ablation längerer Impulsabgabe [11]. Ziel einer neuen Ablationsmethode mittels kurzer, hochenergetischer Impulse, der sog. $\operatorname{HPSD}($,high power short duration“)Ablation, ist es daher, im Gegensatz zur konventionelleren Ablation eine effektivere und dabei sicherere Läsionsgeometrie zu erzeugen. Die flachere Eindringtiefe soll hierbei tiefer liegende Strukturen, v.a. den Ösophagus, vor thermischen Schäden schützen [11]. Invitro-Versuche zeigten hier bereits vielversprechende Ergebnisse: Nach Abgabe eines kurzen, aber hochenergetischen
Ablationsimpulses entsteht eine breite, aber flache myokardiale Läsion, optimal z. B. für die Ablation im dünnwandigen linken Vorhof [12].

Ein zusätzlicher Unterschied besteht zudem im Wechsel des Ablationsmodus. Statt der traditionellen leistungsgesteuerten Ablation erlauben moderne HPSDAblationskatheter trotz Spülung der Katheterspitze eine Messung der lokalen Gewebetemperatur und somit eine temperaturgesteuerte Ablation (s. • Abb. 1) [13]. Ob die HPSD-Ablation tatsächlich effektiver und mit weniger Komplikati- 

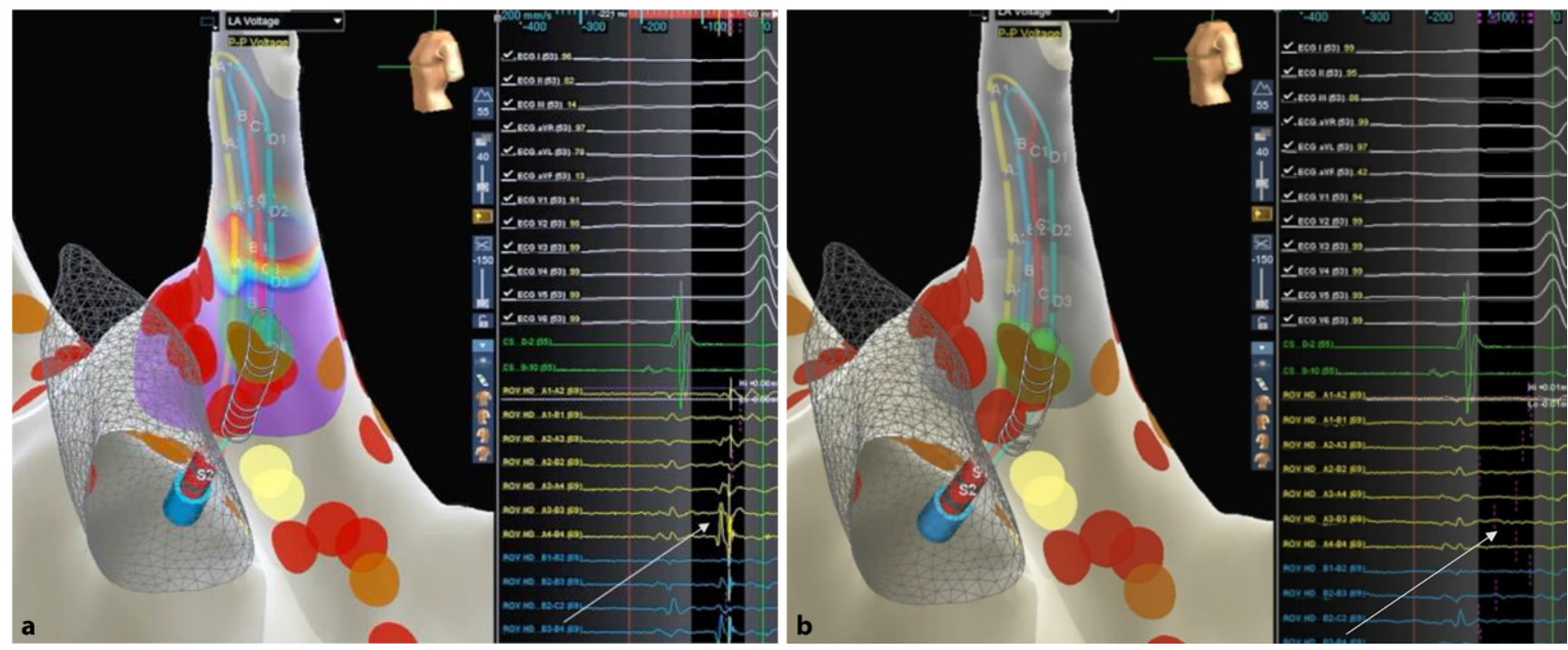

Abb. 1 ॥ Farbkodierter Nachweis des Blocks der Pulmonalvenen während HPSD(,high power short duration“)-Ablation.a Ablationskatheter anterior der linken oberen Pulmonalvene (PV). Die PV-Leitung ist an dem mit dem Pfeil markierten Elektrogramm und der violetten Farbgebung im Map zu erkennen. b Nach Ablation ändert sich bei der Isolation (kein PV-Signal, Pfeil) die Farbgebung auf grau als Hinweis auf die elektrische Isolation der Pulmonalvene.

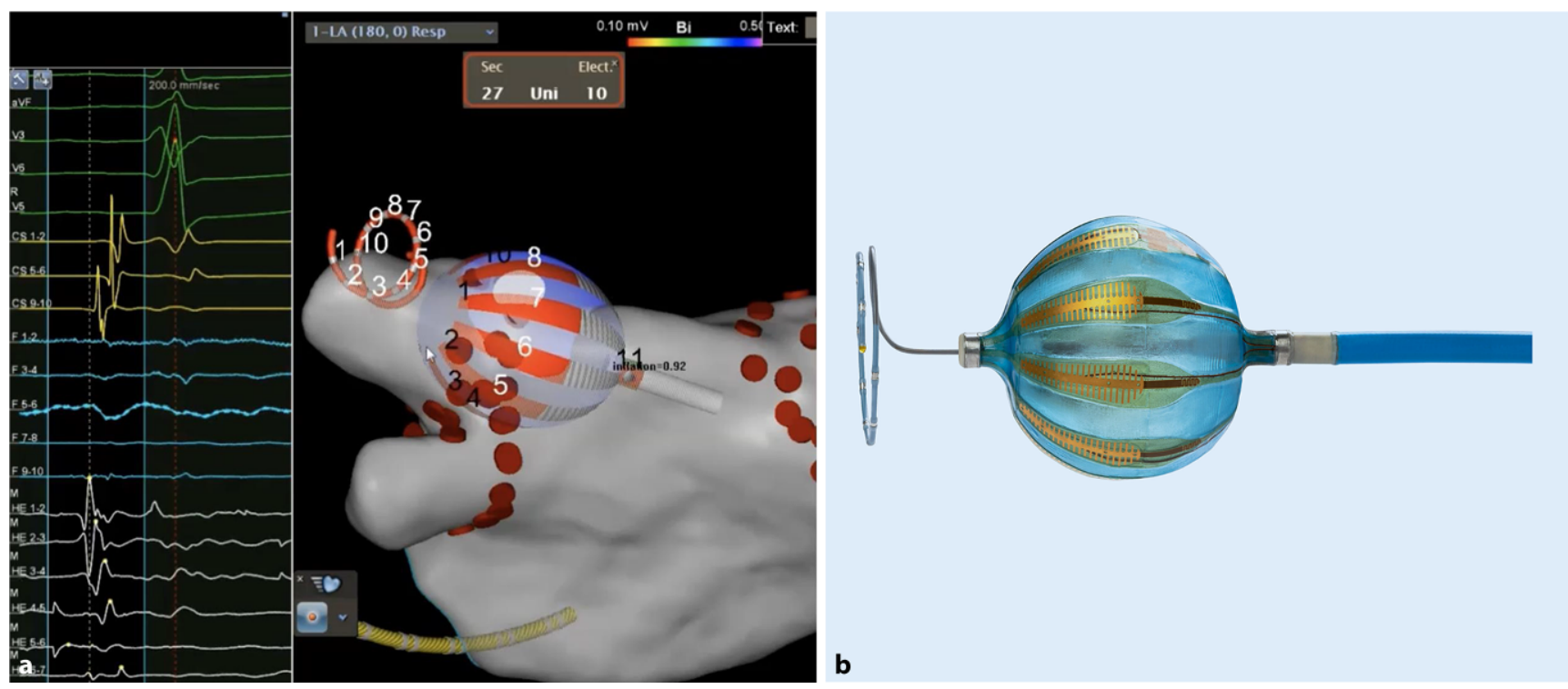

Abb. $2 \Delta$ „Single shot" mittels Hochfrequenzstrom: Mit dieser neuen Technologie können möglicherweise die Vorzüge der schnellen Single-shot-Therapie mit denen des dreidimensionalen Mappings kombiniert werden. Der Ablationsballon lässt sich erstmals in der anatomischen Rekonstruktion integrieren (a). Der gekühlte RF-Ballon mit dem diagnostischen Spiralkatheter im Detail (b). Mit freundlicher Genehmigung von Johnson \& Johnson Medical GmbH

onen verbunden ist, müssen in Zukunft größere randomisierte Studien zeigen. Metaanalysen der bereits vorhandenen Daten zeigen aber jetzt schon eine deutliche Reduktion von Ablationszeiten sowie Prozedurzeiten, und daher hält die Technologie, auch wenn weiterhin weder eine einheitliche Nomenklatur für den "High power short duration“Begriff noch ein einheitliches Protokoll vorliegen, zunehmend Einzug in den klinischen Alltag [14]. Und so erhielten neben der laufenden Nutzung bereits vorhandener Ablationskatheter und Generatoren im vergangenen Jahr 2 Hersteller eine Zulassung für spezielle HPSD-Ablationskatheter. Neben dem QDOT MICRO $^{\circledR}$-Katheter (Biosense Webster Inc., Irvine, CA, USA), der HPSD-Ablation mit $90 \mathrm{~W}$ für $4 \mathrm{~s}$ (Reduktion der Energie ab $65^{\circ} \mathrm{C}$ Gewebetemperatur) ermöglicht, wurden im vergangenen Jahr ebenfalls erste Prozeduren der klinischen Routine mit dem DiamondTemp ${ }^{\text {mw }}$-Katheter (Medtronic, Inc., Dublin, Irland) durchgeführt. Vorgesehen für den DiamondTemp ${ }^{\mathrm{m}}$ ist ein temperaturgesteuertes Protokoll mit $50 \mathrm{~W}$ für $10 \mathrm{~s}$ und einer Zieltemperatur im Gewebe von $50^{\circ} \mathrm{C}$ bis maximal $60^{\circ} \mathrm{C}$. Die mit Industriediamanten bestückte Katheterspitze ermöglicht eine insbesondere unter Ablation artefakt- 
freie Darstellung des Lokalpotenzials. Eine Anpressdruckmessung fehlt in diesem Ablationskatheter, daher werden Zieltemperatur und Attenuierung des Lokalpotenzials als Surrogat für Wandkontakt und Läsionsqualität herangezogen. Darüber hinaus läuft seit Juni 2020 die Zulassungsstudie (ClinicalTrials.gov Identifier: NCT04356040) für einen weiteren dritten Katheter (Tactiflex $^{\mathrm{Tw}}$, Abbott, Plymouth, MN, USA) zur Ablation mit „high power short duration“. Weitere randomisierte Studien müssen demnächst zeigen ob in diesem Verfahren tatsächlich die Zukunft der Hochfrequenzstromablation liegt.

\section{Neue Ablationstechniken - ein Radiofrequenzstrom- Ablationsballon}

Kürzere Ablationszeiten im linken Vorhof und Prozedurzeiten insgesamt sowie einfache Handhabung führten in den letzten Jahren zu einer immer weiteren Verbreitung von Single-Shot-Ablationsverfahren, v. a. des Kryoballons, für die Pulmonalvenenisolation [15]. Verzichtet wird hierbei jedoch auf die Möglichkeit von 3-D-Mapping bzw. auf die Möglichkeit, wenn nötig, patientenindividualisierte Therapien realisieren zu können.

Ein neuer Multielektroden-Radiofrequenzstrom-Ablationsballon (HELIO$\mathrm{STAR}^{\circledR}$, Biosense Webster Inc.) (s. - Abb. 2) soll nun die Vorteile eines Single-Shot-Devices mit der Möglichkeit von 3-D-Mapping verbinden. Der 28-mm-Ballon HELIOSTAR ${ }^{\circledR}$ ähnelt von den Maßen (13,5 French, $28 \mathrm{~mm}$ Durchmesser) dem bekannten Kryoballon. Auf der Oberfläche des Ballons befinden sich jedoch 10 zirkulär angeordnete goldbeschichtete Elektroden, die nach Okklusion der Pulmonalvene eine zirkuläre Hochfrequenzstromläsion um das Pulmonalvenenostium erzeugen können. Jede Elektrode kann dabei einzeln durch Impedanzmessung und Elektrogrammableitung den jeweiligen Wandkontakt anzeigen. Die Temperatur unter Ablation wird zudem von jeder Elektrode einzeln gemessen. So können automatisch bei Übersteigen des Temperaturlimits einzelner Elektroden diese separat reguliert werden.
Durch Integration in das $\mathrm{CARTO}^{\circledR}$ 3-D-Mapping-System (Biosense Webster Inc.) kann zudem ein 3-D-Map über den Ballon erstellt werden [16].

Erste im Januar 2020 veröffentlichte Daten (RADIANCE-Studie [16]) zeigten neben dem vielversprechenden Potenzial dieser Technologie jedoch auch bekannte Probleme. So trat neben vereinzelten asymptomatischen Ösophaguserythemen bei 2,6\% der Patienten eine dauerhafte Parese des N. phrenicus während Ablation an den rechten Pulmonalvenen auf [16]. Hier werden in Zukunft weitere Daten zum Sicherheitsprofil, insbesondere im Hinblick auf Paresen des N. phrenicus benötigt. In 2020 wurde der Ballon auch schon in ersten deutschen Zentren eingesetzt, sodass uns hier für das kommende Jahr viele spannende Erkenntnisse zu dieser neuer Ablationstechnologie erwarten.

\section{Neue Strategien in der Therapie von persistierendem Vorhofflimmern - ein Ausblick}

Eine der schwierigsten Herausforderungen der Vorhofflimmertherapie bleibt auch weiterhin die Behandlung von symptomatischem persistierendem Vorhofflimmern. Neben den pulmonalvenösen Triggern für Vorhofflimmern fanden sich zwar bereits Hinweise, dass atriale Fibrose, dargestellt mittels LateEnhacement in der Magnetresonanztomographie (MRT), zu vermehrten Vorhofflimmerrezidiven führt (DECAAF Study [17]). Diese fibrotischen Areale stellen damit ein mögliches Substrat für persistierendes Vorhofflimmern dar, jedoch konnte bisher kein Vorteil für ausgedehntere linksatriale Ablation z. B. von komplex fraktionierten atrialen $\mathrm{Si}$ gnalen (CFAE) oder Anlage linearer Läsionen gegenüber der reinen Pulmonalvenenisolation gezeigt werden (STAR AF II-Studie [18]). Auch spezielle Mappingstrategien und Katheter z.B. zum Mapping fokaler Impulse und Rotoren (FIRM) oder Dipole-Density-Mapping mittels speziellen Ultraschall-gestützten Mappingkatheters (AcQMap ${ }^{\circledR}$, ACUTUS Medical etc., Carlsbad, CA, USA) zur Visualisierung der atrialen Aktivierung während Vorhofflimmern zeigten zwar in vereinzelten, v. a. nicht randomisierten Studien anfangs vielversprechende Ergebnisse, fanden aber bisher keine breite Anwendung in der klinischen Routine. So blieb auch im Jahr 2020 in den neuen Leitlinien jede Vorhofflimmerablation über die Pulmonalvenenisolation hinaus lediglich eine Klasse-IIb-Empfehlung [9].

Neue Daten und Behandlungsansätze sind hier demensprechend dringend nötig: Bereits Ende 2019 war der Einschluss in die DECAAF II-Studie (ClinicalTrials.gov Identifier: NCT01150214) erfolgreich beendet worden. DECAAF II soll im Hinblick auf linksatriales Substrat die Frage beantworten, ob eine gezielte $\mathrm{Ab}$ lation von MRT-morphologisch identifizierter linksatrialer Fibrose einen neuen möglichen Behandlungsansatz darstellen könnte. Insgesamt gewinnt auch mit den neuen Leitlinien die Substratcharakterisierung immer weiter an Relevanz.

$\mathrm{Ob}$ die elektrische Isolation des Vorhofohres als mögliches arrhythmisches Substrat einen Vorteil bietet, wird derzeit in verschiedenen, auch in Deutschland initiierten Studien untersucht (ASTRO AF, NCT04056390). Hier wird bei Patienten mit festgestellter dauerhafter Isolation der Pulmonalvenen und dennoch dokumentiertem $\mathrm{AF}$ („atrial fibrillation“)-Rezidiv randomisiert entweder mittels konventioneller Ablation oder mittels elektrischer Vorhofohrisolation behandelt. Darüber hinaus läuft bereits seit Dezember 2019 der Einschluss in eine weitere Studie zur Evaluation der Bedeutung einer ergänzenden Vorhofohrisolation im Rahmen der Kryoablation bei persistierendem oder lang persistierendem Vorhofflimmern (LALA-Land-AF, ClinicalTrials.gov Identifier: NCT04240366). Für die kommenden Jahre erwarten uns also spannende neue Einblicke und dringend benötigte Antworten auf die Frage nach der optimalen Therapie von persistierendem Vorhofflimmern.

\section{Fazit für die Praxis}
- Drei neue Landmark-Trials unterstrei- chen nicht nur die Effektivität der interventionellen Vorhofflimmerbe- handlung, sondern zeigen erstmals


einen prognostischen Vorteil der Rhythmuskontrolle.

- Die neue europäische Leitlinie für Diagnostik und Therapie von Vorhofflimmern stellt eine dezidierte Charakterisierung des Vorhofflimmerns in den Vordergrund und weitet die Indikation der Katheterablation aus.

- Neue vielversprechende Ablationsverfahren erreichten im vergangenen Jahr Marktreife oder stehen kurz davor. Neue Daten, insbesondere zur immer wichtiger werdenden Substratcharakterisierung, stehen kurz vor Veröffentlichung, sodass uns für das kommende Jahr spannende neue Erkenntnisse erwarten.

\section{Korrespondenzadresse}

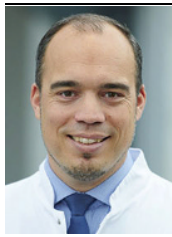

Philipp Sommer

Klinik für Elektrophysiologie und Rhythmologie, Herz- und Diabeteszentrum NRW, RuhrUniversität Bochum Georgstr. 11, 32545 Bad Oeynhausen, Deutschland psommer@hdz-nrw.de

\section{Einhaltung ethischer Richtlinien}

Interessenkonflikt. D. Steven: Studienunterstützung und Vortragshonorare von Abbott, Medtronic, Biosense Webster und Boston Scientific. P. Sommer: Mitglied des Advisory Boards von Abbott, Medtronic, Biosense Webster und Boston Scientific. J.-H. van den Bruck gibt an, dass kein Interessenkonflikt besteht.

Für diesen Beitrag wurden von den Autoren keine Studien an Menschen oder Tieren durchgeführt. Für die aufgeführten Studien gelten die jeweils dort angegebenen ethischen Richtlinien.

\section{Literatur}

1. Marijon E, Le Heuzey JY, Connolly S et al (2013) Causes of death and influencing factors in patients with atrial fibrillation: a competing-risk analysis from the randomized evaluation of longterm anticoagulant therapy study. Circulation 128(20):2192-2201

2. Van Gelder IC, Hagens VE, Bosker H et al (2002) A comparison of rate control and rhythm control in patients with recurrent persistent atrial. Fibrillation 347(23):1834-1840

3. Magnussen C, Niiranen TJ, Ojeda FM et al (2017) Sex differences and similarities in atrial fibrillation epidemiology, risk factors, and mortality in community cohorts: Results from the biomarcare consortium (Biomarker for cardio- vascular risk assessment in Europe). Circulation 136(17):1588-1597

4. Wyse D, Waldo AL, DiMarco J et al (2002) Comparison of rate control and rhythm control fo atrial fibrillation. N Engl J Med 346(5):305-310

5. Packer DL, Mark DB, Robb RA et al (2019) Effect of catheter ablation vs antiarrhythmic drug therapy on mortality, stroke, bleeding, and cardiac arrest among patients with atrial fibrillation: the CABANA randomized clinical trial.JAMA321(13):1261-1274

6. Kirchhof P, Camm AJ, Goette A et al (2020) Early rhythm-control therapy in patients with atrial fibrillation. NEngl J Med 383(14):1305-1316

7. Andrade JG, Wells GA, Deyell MW et al (2020) Cryoablation or drug therapy for initial treatment of atrial fibrillation. N Engl J Med 384(4):305-315. https://doi.org/10.1056/NEJMoa2029980

8. Wazni OM, Dandamudi G, Sood N et al (2020) Cryoballoon ablation as initial therapy for atrial fibrillation. N Engl J Med 384(4):316-324. https:// doi.org/10.1056/NEJMoa2029554

9. Hindricks G, Potpara T, Dagres N et al (2020) 2020 ESC Guidelines for the diagnosis and management of atrial fibrillation developed in collaboration with the European Association for Cardio-Thoracic Surgery (EACTS). Eur Heart J Oxford University Press (OUP) :1-126

10. Willems S, TilzRR, Steven D, KääbS, Wegscheider K, Gellér L, Meyer C, Heeger CH, Metzner A, Sinner MF Schlüter $\mathrm{M}$, Nordbeck $\mathrm{P}$, Eckardt L, Bogossian $\mathrm{H}$, Sultan A, Wenzel B, Kuck KH (2020) BERLIN VT Investigators. Preventive or Deferred Ablation of Ventricular Tachycardia in Patients With Ischemic Cardiomyopathy and Implantable Defibrillator (BERLIN VT): A Multicenter Randomized Trial. Circulation 141(13):1057-1067. https://doi.org/ 10.1161/CIRCULATIONAHA.119.043400

11. Leshem E, Zilberman I, Tschabrunn CM et al (2018) High-power and short-duration ablation for pulmonary vein isolation: biophysical characterization. JACC Clin Electrophysiol 4(4):467-479

12. Bourier F, Martin CA, Takigawa M et al (2018) High - power short-duration versus standard radiofrequency ablation : Insights on lesion metrics. J Cardiovasc Electrophysiol 29(11):1570-1575. https://doi.org/10.1111/jce.13724

13. Iwasawa J, Koruth JS, Petru J et al (2017) Temperature-controlled radiofrequency ablation for pulmonary vein isolation in patients with atrial fibrillation. J Am Coll Cardiol 70(5):542-553

14. Kewcharoen J, Techorueangwiwat C, Kanitsoraphan C et al (2020) High-power short duration and low-power long duration in atrial fibrillation ablation: a meta-analysis. J Cardiovasc Electrophysiol. https://doi.org/10.1111/jce.14806

15. Kuck KH, Brugada J, Fürnkranz A et al (2016) Cryoballoon or radiofrequency ablation for paroxysmal atrial fibrillation. $\mathrm{N}$ Engl J Med 36(5):393-394

16. DhillonGS,HonarbakhshS, DiMonaco Aetal(2020) Use of a multi-electrode radiofrequency balloon catheter to achieve pulmonary vein isolation in patients with paroxysmal atrial fibrillation: 12-Month outcomes of the RADIANCE study. JCardiovasc Electrophysiol 31(6):1259-1269

17. Marrouche NF, Wilber $D$, Hindricks $G$, Jais $P$, Akoum N, Marchlinski F, Kholmovski E, Burgon N, Hu N, Mont L, Deneke T, Duytschaever M, Neumann T (2015) Association of atrial tissue fibrosis identified by delayed enhancement MRI and atrial fibrillation catheter ablation the DECAAF study. JAMA 84132(5):498-506

18. Verma A, Chen J, Deisenhofer I et al (2018) Approaches to catheter ablation for persistent atrial fibrillation. New Engl J Med 372(19):1812-1822

\section{Aus dem Takt}

Ein Forschungsteam der FriedrichAlexander-Universität ErlangenNürnberg (FAU) hat ein Verfahren entwickelt, mit dem Vitalfunktionen per Radar zuverlässig detektiert und diagnostiziert werden können. In einer neuen Studie zeigen die Forschenden nun, dass das Radar auch die Herzratenvariabilität (HRV) zuverlässig messen kann. Das Radar könnte zukünftig durch Langzeitbeobachtungen der HRV pathologische Veränderungen frühzeitig erkennen und so schweren Krankheitsverläufen vorbeugen.

Das menschliche Herz schlägt nicht gleichmäßig wie ein Metronom, sondern variiert je nach Gefühlslage der zugehörigen Person: Stress, Schmerzen und Depressionen können die Herzratenvariabilität(HRV) verändern. Aber auch chronische Krankheiten wie Diabetes und Herzerkrankungen wirken sich auf die HRV aus.

Um die HRV mit dem Radar zu erfassen, hat das Team der FAU und der Technischen Universität Hamburg die Radartechnik mit Methoden des maschinellen Lernens kombiniert. Im Rahmen eines klinischen Experiments am Universitätsklinikum Erlangen konnte das Team die Zuverlässigkeit des Verfahrens bestätigen.

Beim sogenannten Eiswasser-Test halten die Testpersonen ihre Hand für eine bestimmte Zeit in Eiswasser, so dass eine Schmerzreaktion ausgelöst wird, die zu einer abrupten Änderung der HRV führt. Diese Änderung zeichnete das Team sowohl mit dem Radar als auch mit einem EKG als Referenz auf. Beim Vergleich zeigte sich eine sehr hohe Übereinstimmung zwischen den Methoden.

Neben dem potenziellen Einsatz zur Beobachtung von Patient*innen im Krankenhaus, etwa um eine Sepsis oder einen plötzlichen Herzstillstand frühzeitig erkennen zu können, könnte das Verfahren auch im heimischen Bereich eingesetzt werden, um eine Langzeitbeobachtung der HRV durchzuführen. 